\title{
Hybrid Approach to Implement Multi-robotic Navigation System Using Neural Network, Fuzzy Logic and Bio-inspired Optimization Methodologies
}

Shahanaz Ayub ( $\sim$ sayub_bietjhs@rediffmail.com )

BIET Jhansi: Bundelkhand Institute of Engineering and Technology

Navneet Singh

Veer Kunwar Singh University

Md. Zair Hussain

Maulana Azad National Urdu University

Mohd Ashraf

Maulana Azad National Urdu University

Dinesh Kumar Singh

Dr Shakuntala Misra National Rehabilitation University

Anandakumar Haldorai

Sri Eshwar College of Engineering

\section{Research Article}

Keywords: Bio-inspired, Particle Swarm Optimization (PSO), Fuzzy logic, Neural Network, Multi-robot Navigation

Posted Date: October 18th, 2021

DOl: https://doi.org/10.21203/rs.3.rs-975723/v1

License: (c) (i) This work is licensed under a Creative Commons Attribution 4.0 International License. Read Full License 


\title{
HYBRID APPROACH TO IMPLEMENT MULTI-ROBOTIC NAVIGATION SYSTEM USING NEURAL NETWORK, FUZZY LOGIC AND BIO-INSPIRED OPTIMIZATION METHODOLOGIES
}

${ }^{1}$ Dr. Shahanaz Ayub, ${ }^{2}$ Mr. Navneet Singh, ${ }^{3}$ Dr. Md. Zair Hussain, ${ }^{4}$ Dr. Mohd Ashraf, ${ }^{5}$ Dr. Dinesh Kumar Singh, 6 Dr. Anandakumar Haldorai

1 Associate Professor, Electronics and Communication Engineering Department, Bundelkhand Institute of Engineering and Technology, Jhansi, Uttar Pradesh, Pin-284128, sayub_bietjhs@rediffmail.com

2 Assistant Registrar, Veer Kunwar Singh University, Ara, Bihar, India. navneetsvu@ gmail.com

3 Associate Professor, Information Technology, School of Technology, Maulana Azad National Urdu University, Hyderabad (TS) mdzairhussain@gmail.com

4 Associate Professor, Computer Science \& Engineering, School of Technology, Maulana Azad National Urdu University, Hyderabad (TS) ashraf.saifee@gmail.com

5 Assistant Professor, Department of IT, DSMNRU, Lucknow, UP, India. dineshsingh025@gmail.com

6 Associate professor, Department of Computer science and engineering, Sri Eshwar College of Engineering, Coimbatore, Tamil Nadu, India-641202 anandakumar.psgtech@gmail.com

\begin{abstract}
Mobile robots have been increasingly popular in a variety of industries in recent years due to their ability to move in variable situations and perform routine jobs effectively. Path planning, without a dispute, performs a crucial part in multi-robot navigation, making it one of the very foremost investigated issues in robotics. In recent times, meta-heuristic strategies have been intensively investigated to tackle path planning issues in the similar way that optimizing issues were handled, or to design the optimal path for such multi-robotics to travel from the initial point to such goal. The fundamental purpose of portable multi-robot guidance is to navigate a mobile robot across a crowded area from initial point to target position while maintaining a safe route and creating optimum length for the path. Various strategies for robot navigational path planning were investigated by scientists in this field. This work seeks to discuss bio-inspired methods that are exploited to optimize hybrid neuro-fuzzy analysis which is the combination of neural network and fuzzy logic is optimized using the Particle Swarm optimization technique (PSO) in realtime scenarios. Several optimization approaches of bio-inspired techniques are explained briefly. Its simulation findings, which are displayed for two simulated scenarios reveal that hybridization increases multi-robot navigation accuracy in terms of navigation duration and length of the path.
\end{abstract}

Keywords: Bio-inspired, Particle Swarm Optimization (PSO), Fuzzy logic, Neural Network, Multi-robot Navigation. 


\section{INTRODUCTION}

Physical chores that were formerly regarded to be undertaken by humans are now undertaken by robotics to reduce the load of labor on humankind. Nonetheless, there seems to be a requirement that robotics not only perform physical activities but also analyze and make decisions in the same way that humans do. Artificial intelligence understanding is vital in making things smart and achieving this goal. Path design is the most prevalent difficulty in multi-robot guidance; robots must navigate from a beginning location to the desired position while avoiding impediments. Several approaches have been explored on robotics such as the mini air vehicles [1], wall-climbing automaton [2,3], mobility robotic, submerged robotics, and mobility robot.

A multi-objective scheduling issue for wheeling movable robots must be solved using stochastic particulate swarm optimization (PSO) as well as the firefly approach [4]. Artificial Intelligence (AI) seems to be the study of recognizing and creating something smart, which aids in creating excellent decisions and completing tasks. To resolve issues, a smart system needs specific strategies, which are known as Technologies based on Artificial Intelligence. Various AI approaches are well recognized, including Fuzzy logic systems, Neuro-Fuzzy, Neural Networks (NN), and Computational Approaches such as Genetic Algorithms (GA), Particles Swarm Optimizing, Bio-inspired Computational Strategies, and Ant Colony Optimization (ACO).

Currently, numerous bio-based optimization techniques can be discovered in research; it is estimated that they are greater than 150 various strategies and enhanced strategies for choosing the optimum outcomes on optimal issues. Even though we have done work with various techniques in various aspects, such as, parameter modification that uses fuzzy logic and neural network, the identified methodologies are picked since they have proved to become a great option for attempting to solve several optimization issues, with actual methodologies, and we also have the knowledge required to work with each other. Instead, we concentrated on applications including an optimized neuro-fuzzy approach. The article provides applications using various optimizing approaches, vital data about related themes, and so on which are employed by investigators in this field. It is important to note that they are numerous technologies provided which can be employed to gather data and afterward examine any area of research. We may see the graphical depiction in various methods in a matter of moments which helps research a specific issue. The research establishment can profit from learning about and promptly obtaining subjects, writers, nations, journal kinds, years, and so on from all over the globe. This is important since, despite the high number of referrals and saved records, we may utilize the technologies to autonomously design networking using clusters and nodes. Lastly, to find out, for instance, who is researching on a specific subject or which author has received the most references in a given year. For this instance, we concentrated solely on bio-inspired optimizing namely Particle Swarm optimization of hybrid neuro-fuzzy analysis. With such a concept, though, numerous writers can utilize various ways to retrieve valuable material about every field of research and industry. 


\section{RELATED WORK}

For improving robotic guidance effectiveness, [5] combined fuzzy logic and flexible computational approaches such as PSO, NN, and GA for optimizing the various membership module variables. They demonstrated two fuzzy logic habits: movement to targets (MFLC) and impediment prevention behavior (AFLC). Fuzzy Pulse Width Modulation (PWM) controllers are described in [6]. In [7], a genetic-neural, as well as genetic-fuzzy controller for adaptable navigational path modeling of such a robotic system among dynamical barriers, is developed. GA was utilized in their study to change the strength fuzzy association function and also NN. A fuzzy logic controller for mobile robots in densely congested impediment environments must be created [8]. Goal-seeking and impediment minimization are two behavioral controllers developed by the researchers. The magnetic measuring approach is being used to assess goal-seeking attitude, while the ranges finding device is used to assess impediment avoiding behavior. A genetic-fuzzy approach based upon fuzzy logic and GA is proposed to address the robotic motion issue in a complex world [9]. An evolutionary technique for optimizing the preceding and subsequent variables of a fuzzy controller must be devised and deployed [10]. A sensing wireless fuzzy controller is created for enabling mobile robots among active and passive elements in sectors [11]. Tracker fuzzy logic control (TFLC) and Obstacle avoidance fuzzy logic controller (OAFLC) are two separate fuzzy controllers that assist the robots in finding a collision-free route to their goal. An action-based fuzzy logic controller is proposed to handle the challenge of mobile robots in an uncertain place [12]. A variety of fuzzy-rule-based controllers assist the robots in escaping from a bind and predicting their attitude. The four distinct logic controllers for vehicle mobile robots are wall follower fuzzy, paralleled parking fuzzy, vault parking fuzzy, cornering control fuzzy, vault parking fuzzy, and paralleled parking fuzzy [13]. The developed fuzzy controller is verified in actual time on such an FPGA. A real-time fuzzy monitoring controller for robot navigation targets using infrared sensors [14]. Introduced a smart agent-based fuzzy logic structure that lets the robots travel on its self in an unpredictable domain without human interaction [15]. The path modeling technique using a fuzzy controller is created [16].

Yang and Zhu suggested the reactive-based neuro-fuzzy navigational strategy for portable robots [17]. This approach was used to create two activities, impediment detection, and target seeking, as well as 48 rules. An NN-based method is implemented to define the values of the objective function that lowers the navigational route duration from beginning to finish point. Regional-based functions neural network (RBFNN), as well as Takagi-Sugeno-type controllers, are used to resolve the robotic arm path tracking issue [18]. Whereas NN adjusts the values of the basic functions, fuzzy logic deals with the ambiguity of the surroundings. A sensor-based guidance system that is depending on a neuro-fuzzy structure that utilized eight ultrasound-ranging detector sensors to identify obstructions is developed [19]. The authors combined neuro-fuzzy structure to behaviorbased design towards mobile robots in an uncertain place. The NN has been designed to retrain the robots to achieve the destination while the fuzzy logic controller is utilized to manage the robot's motions [20]. 
An impediment avoidance approach is suggested for wheeled robot navigation position stabilization utilizing periodic type-2 fuzzy NN controllers [21]. A RAM-based neuro-fuzzy technique for mobile robots was described [22]. The authors employed the NN for managing the robot's bearing angle while moving and a fuzzy controller for analyzing the sensor data. The neurofuzzy detector is developed for controlling the steering of a robotic in such an unfamiliar area [23]. The combination of softer computational approaches such as NN as well as fuzzy inferences systems increases the robot's decision-making performance as well as learning in such uncertain surroundings [24]. Integrated neuro-fuzzy controllers for movable robot guidance amongst various impediments were created [25]. The Adjustable Neuro Interpretation System (ANFIS) approach is employed to solve the nonlinear control wheeled robotic path issue [26]. The authors used the steepest gradient training approach to modify the values of the membership functions. The writers created two control systems: an Artificial Neural Networks (ANN) for the robotic ceiling as well as a fuzzy controller enabling obstacle minimization [27]. Whenever the robots move towards their targeted goal, the controllers receive data from the various sensors for avoiding impediments in the path. By incorporating acoustic detectors with NN design, Wang and Zhao addressed the navigational issue for independent robots [28].

\section{HYBRID MODEL FOR MULTI-ROBOT NAVIGATION}

\subsection{Fuzzy Logic:}

The idea of fuzziness must be developed as a derivation from a common set. Fuzzy rules, as opposed to common sets, include constituents with varying membership degrees. The chance of a component is to the collection is referred to as members. The triangle objective function was employed for path selection in this research. The fuzzy inference system (FIS) is an essential component of the fuzzy logic system. The hypothesized instead of traditional strict models; advanced methods may be regulated employing fuzzy logic. To demonstrate the logic characterized by the objective function, a fuzzy control employs conditional phrases known as deductions. The fuzzy logic system is depicted in Figure 1. At first, the crisp integers are used as inputs. FIS's three fundamental elements are (i) fuzzification, (ii) defuzzification (iii) fuzzy illusion engine.

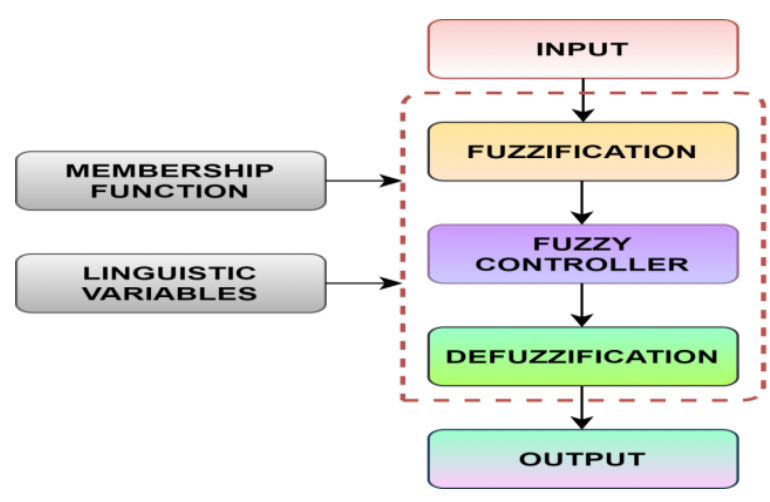

Figure 1: Fuzzy logic controller. 
Fuzzification: The method of converting a sharp number to such a fuzzy linguistic is known as fuzzification. The objective function is used to carry out this modification. Under this work, triangle participation functions are applied, as seen in Figure 1. Every linguistic variable's fuzzy data element will be specified in its discursive world. The participation function characterizes the fuzzy parameters with all values in $(0,1)$. The fuzzy rules for this research are comparative distance to a destination, angle towards its destination, and proximity to the closest impediment. Every linguistics variable's fuzzy parameter set comprises (medium, low and higher).

Fuzzy Illusion Engine: It is the most important part of such a fuzzy logic system. The fuzzy control engine's function is to create conclusions based on If...then principles. The principles are expressed as follows:

RULE I: if $D$ is $a_{1}$ and $E$ is $b_{1}$, then $F$ is $c_{1}$

RULE II: if $D$ is $a_{2}$ and $E$ is $b_{2}$, then $F$ is $c_{2}$

RULE N: if $D$ is $a_{i}$ and $E$ is $b_{i}$, then $F$ is $c_{i}$

Where $\mathrm{D}$, as well as $\mathrm{E}$, is dependent elements, $\mathrm{F}$ is the responder parameter and $a_{i}, b_{i}, c_{i}$ are triangle membership functions-defined fuzzy variables. After the selections are established a fuzzy logic controller is utilized to analyze the rules' conclusions. The Mamdani fuzzy logic controller is employed within that research for rule analysis as described by,

RULE I: $\mu_{1}=\mu_{a 1}(d) \wedge \mu_{b 1}(e)$

RULE II: $\mu_{2}=\mu_{a 2}(d) \wedge \mu_{b 2}(e)$.

RULE N: $\mu_{i}=\mu_{a i}(d) \wedge \mu_{b i}(e)$

Defuzzification: During the defuzzification process, the fuzzy parameters are turned to crisper outputs. A centric approach is utilized for defuzzification within that work. The Euclidean method's defuzzified result $\mathrm{x} *$ could be expressed as follows.

$x^{*}=\frac{\int \mu_{A}(x) x d x}{\int \mu_{A}(x) d x}$

\subsection{Neural Network:}

Artificial neural networks (ANNs) are smart systems made up of numerous basic and strongly coupled functional units. Such elements transmit data due to their flexibility to respond dynamically to exterior inputs. This $\mathrm{NN}$ is represented by mostly well-organized levels of interconnecting nodes. These nodes are made up of an activating factor. Such structures then interact with buried layers for real analysis through a balanced linkage scheme. To provide the necessary response, the buried layers communicate well with output nodes. The generalization capability, dispersed representations, enormous concurrency, learning capabilities, 
and error endurance of $\mathrm{NN}$ make it valuable in the area of movable multi-robot navigation. Usage of NN for the guidance of such a wheeled multi-robot navigation is largely unidentified surroundings, as well as the employment of dual NN-based methods for the generation of the collision-free route. The initial neural method uses perceptual input to detect space, while another NN determines a secure track by evading the closest impediment. To prevent human guiding during the navigational operation an autonomous learning technique was proposed. Their approach distinguishes itself in that, depending upon the intricacy of the surroundings; the NN modifies the addition and removal of new buried layers while learning without human intervention to complete the navigational job. The use of NN in Rapid Synchronous Localization, as well as Mapping approach (Fast SLAM), is demonstrated to reduce the fault buildup caused by improper trajectory models and incorrect quantization of such SLAM asymmetric functions. The combination of NN with Quick SLAM improves the portable robot's ability to traverse in an uncertain place avoid colliding with obstacles. To attain the best possible outcome for a multi-robot navigational task,

\subsection{Development of a hybrid Neuro-fuzzy network:}

Smart soft computational methods, including artificial neural network (ANN) and fuzzy logic, have recently been proven to be effective and suited for the development of smarter networks. To manage inaccurate and ambiguous networks, a fuzzy logic system relies primarily on the picking and dispersion of association functions as well as its if-then logic is utilized to obtain information from human expertise. The neural networks may learn from their surroundings and evolve in an interacting manner. For such cases, a neural network in conjunction with a fuzzy inference system has resulted in neuro-fuzzy systems that combine the benefits which include both artificial neural networks and fuzzy inference systems.

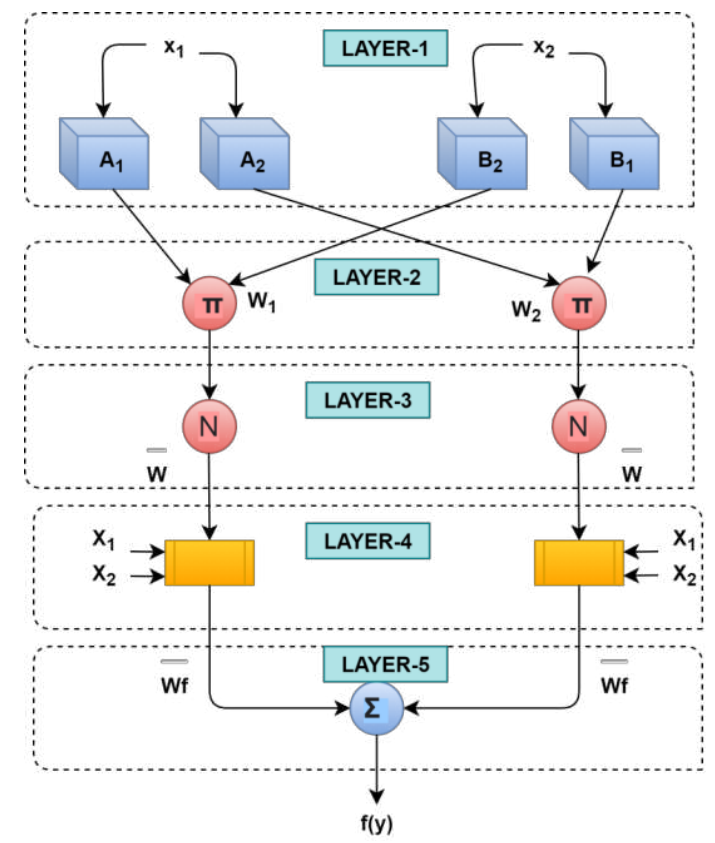

Figure 2: ANFIS structure. 
Consider, for the current ANFIS design, that the fuzzy logic under discussion includes two input data's $x_{1}$ and $x_{2}$ as well as single outcome y, as illustrated in Figure 2.

Assume that fuzzy inferences network has 2 Takagi-Sugeno if-then criteria.

Rule 1: When $x_{1}$ equals $A_{1}$ AND $x_{2}$ equals $B_{1}$, So $y_{1}=p_{1} x_{1}+q_{1} x_{2}+r_{1}$.

Rule 2: When $x_{1}$ equals $A_{2}$ AND $x_{2}$ equals $B_{2}$, Soy $y_{2}=p_{2} x_{1}+q_{2} x_{2}+r_{2}$.

Here, $\mathrm{A}_{1}, \mathrm{~A}_{2}, \mathrm{~B}_{1}$, and $\mathrm{B}_{2}$ are language descriptions of the inputs $x_{1}$ as well as $x_{2} .\left\{p_{1}, q_{1}, r_{1}\right\}$ as well as $\left\{p_{2}, q_{2}, r_{2}\right\}$ are subsequent variables, $y_{1}$ as well as $y_{2}$ are the system's anticipated outputs. The ANFIS architecture consists of five layers, functions of which are detailed in detail below:

Layer-1: This is also referred to as a fuzzification level. This layer's nodes are all adaptable (square nodes) that compute the membership functional results in such a fuzzy system. These nodes' results are computed as follows:

$\left.\begin{array}{l}O_{1, i}=\mu_{A_{i}}\left(x_{1}\right) \\ O_{1, i}=\mu_{B_{i}}\left(x_{2}\right)\end{array}\right] \quad i=1,2$

In many other terms, $O_{1, i}$ are the association functions for $A_{i}$ and $B_{i}$ that indicates the extent to which $x_{1}$ as well as $x_{2}$ satisfy the quantifiers $A_{i}$ and $B_{i}$. Fuzzy association functions can take any structure such as triangles, trapezoid, Gaussian, bell-shaped, and so on. Typically, we select the bell-shaped it having a peak value of 1 and the lowest value of 0 . This bell-shaped function is used to represent the objective function of $\mathrm{A}$ but also $\mathrm{B}$ in this case

$$
\begin{aligned}
& \mu_{A_{i}}\left(x_{1}\right)=\frac{1}{1+\left[\left(\frac{x_{1}-c_{i}}{a_{i}}\right)^{2}\right]^{b_{i}}} \\
& \mu_{B_{i}}\left(x_{2}\right)=\frac{1}{1+\left[\left(\frac{x_{2}-c_{i}}{a_{i}}\right)^{2}\right]^{b_{i}}}
\end{aligned}
$$

where if the bell-fuzzy integers are $a_{i}, b_{i}$, and $c_{i}$. They're sometimes referred to as predecessor or assumption constraints.

Layer-2: These are referred to as the rules layer. These layer's nodes are all fixed nodes (circular nodes). Every rule node gets inputs from the relevant layer-2 nodes and computes the firing intensity of every fuzzy guideline. The layer's outcome is the sum of all receiving impulses (inputs).

$$
O_{2, i}=\omega_{i}=\mu_{A_{i}}\left(x_{1}\right) \cdot \mu_{B_{i}}\left(x_{2}\right)
$$

While $\omega_{i}$ denotes the ith rule's firing intensity. 
Layer-3: This is also the normalization level, and each node in there is a constant point (circular node). The layer's results are referred to as normalized firing intensity. The normalized firing intensity of its ith node is measured by adding up the firing intensity of an ith point to just the total firing strengths of all criteria.

$$
O_{3, i}=\overline{\omega_{l}}=\frac{\omega_{i}}{\omega_{1}+\omega_{2}} \quad i=1,2
$$

The normalized firing intensity of a specific rule is represented by $\overline{\omega_{l}}$.

Layer-4: It's the layer of defuzzification. These layer nodes are all adaptable nodes. Every node is linked to the appropriate normalization node and gets the starting inputs $x_{1}$ as well as $x_{2}$. The defuzzification nodes produce the balanced subsequent meaning of a specified rule, as shown below:

$O_{4, i}=\overline{\omega_{l}}\left(p_{i} x_{1}+q_{i} x_{2}+r_{i}\right) \quad i=1,2$

While $O_{4, i}$ denotes the result of node i in layer- 4 , and $\left(p_{i}, q_{i}, r_{i}\right)$ are the attributes for such nodes. Such attributes are again referred to as subsequent attributes.

Layer-5: A unique summing node represents it. It is a static node, and it is designated as $\sum$. That node computes the total system outcome by adding every defuzzification node from the preceding level.

$$
\begin{aligned}
O_{5, i} & =\sum_{i} \overline{\omega_{l}}\left(p_{i} x_{1}+q_{i} x_{2}+r_{i}\right) \\
& =\frac{\sum_{i} \omega_{i}\left(p_{i} x_{1}+q_{i} x_{2}+r_{i}\right)}{\sum_{i} \omega_{i}} \quad i=1,2
\end{aligned}
$$

$O_{5, i}$ denotes the outcome of node i at layer-5. In ANFIS, the hybrid learned method is used to upgrade all factors (basis and subsequent parameters) to minimize the difference among the ANFIS result and testing set. ANFIS variable adjustment comprises a front and reverses pass. The assumption variables are set in the front pass including its hybrid training approach, the operational signals advance to layer-4, as well as the subsequent variables then are determined using the lowest square estimating (LSE) approach. The subsequent variables are set in the retrograde pass and all fault rates flow backward. The gradient approach is used to upgrade the preceding variables. The steepest descent approach makes it harder to choose the appropriate educational variables, and the variable converging rate is still slow. To address this intricacy issue, we propose a new inherent nature Particle Swarm Optimization (PSO) method for selecting optimum values in the ANFIS. The PSO method will not necessitate a training set and delivers a quicker variable converging rate in the ANFIS. 


\subsection{Neural Network-based Fuzzy logic controller}

This neuro-fuzzy controller is developed for mobility multi-robot guidance in an unorganized environment with the objective function of sources and neuro-fuzzy inference implications that are individually generated. A certain fuzzy controller is utilized to achieve the destination and prevent collisions with barriers by inverting the speeds of two tires. In general, a neuro-fuzzy controller consists of three components: lexical parameters, association functions that reflect the graphical depiction of outputs as well as inputs, and fuzzy sets that include assigning an activity to every multi-robot circumstance. Every controller in this research has 2 inputs: angle (among the robots and the goal or the robotic and the impediment) and distance (among the robots and the destination or the robots and the impediment). The following are the formulas for the assigned distance $\mathrm{d}$ and angle $\varphi$ :

$$
\begin{gathered}
d=\sqrt{\left(x_{T}-x\right)^{2}+\left(y_{T}-y\right)^{2}} \\
\varphi=\theta_{T}-\alpha
\end{gathered}
$$

Where $\quad \theta_{T}=\tan ^{-1} \frac{\left(y_{T}-y\right)}{\left(x_{T}-x\right)}$

Here $(x, y)$ seems to be the exact location of the robotic system, $\left(x_{T}, y_{T}\right)$ seems to be the location of the goal or impediment, and $\theta_{T}$ and $\alpha$ are the orientations of the goal or impediment as well as the robotic arm with regards to the $\mathrm{X}$-axis, correspondingly. For proximity entry, five gaussian objective functions were provided. This input's range is separated into five linguistics factors: Very Big (VB), Small (S), Big (B), Medium (M), and Very Small (VS). Seven objective functions are allocated to the angular input, as well as the extent of such input is divided into seven linguistics factors: Zero (Z), Negative Big (NB), Negative Small (NS), Negative Medium (NM), Positive Big (PB), Positive Small (PS), and Positive Medium (PM).

The fuzzy controller contains output signals, which have been the two-wheel speeds (right and left wheel speeds). The fuzzy logic employed in this research is a Takagi-Sugeno type-0 method. As a result, the outcomes of fuzzy sets are invariant. Such outputs are classified as five linguistic terms: Small (S), Zero (Z), Medium (M), Very Big (VB), and Big (B). The objective functions of source parameters (distance and angle) are depicted, the linguistics variable quantities of outcomes (left and right wheel speeds), the right wheel speed fuzzy system, and the left wheel speed fuzzy system. In the following sections, we will employ the evolutionary algorithms and PSO and type0 fuzzy logic parameters for improving its performance. The membership is one of the parameters that must be fine-tuned the variables of input functions as well as the linguistic parameters of outputs.

\subsection{Particle Swarm Optimization:}

The PSO approach is being used in the section to change the variables (width and center) for input objective function as well as linguistic parameters of outcomes. Particles Swarm Optimizing (PSO) 
is an approach influenced by the activity of animals like bees, birds, fishes, etc. The basic idea behind this optimization strategy is to create a community of particles (solutions) randomly. And in the workplace, every particle does have a location and a speed. Following every repetition, the optimum location of every particle, as well as the globally best place between all component locations, are chosen and designated as 'pbest' as well as 'gbest,' correspondingly. Such two numbers are required to compute the new speeds and locations of the community's particle. Every repetition, the speed, and location of every particle is altered by using the following equations:

$v(t+1)=F \cdot v(t)+k_{1} \cdot r_{1} \cdot($ pbesti $-x(t))+k_{2} \cdot r_{2} \cdot($ gbest $-x(t))$

$p(t+1)=p(t)+v(t+1)$

While $v(t)$ seems to be the real velocity, $v(t+1)$ is the latest velocity, $\mathrm{k} 1$ as well as $\mathrm{k} 2$ are optimistic constants pbest seems to be the particle i's previous preferred place, gbest was its particle's absolute perfect location, while $\mathrm{F}$ seems to be the mass. A chromosome utilized is determined by the values of the input objective function (center and width) as well as the outcomes of the rule base. The fitness value is the proximity between the robots and the objective.

\subsection{Hybrid model for multi-robot navigation:}

The primary goal of this study is to anticipate the appropriate steering angle (SA) of mobile robots for them to travel securely in surroundings utilizing a PSO-based ANFIS controller. We will imagine that the adaptable neuro-fuzzy guidance system under examination contains four sensing input data, like right obstacle distance (ROD) $\left(x_{2}\right)$, Front obstacle distance (FOD) $\left(x_{1}\right)$, heading angle $\left(x_{4}\right)$, Left Obstacle distance (LOD) $\left(x_{2}\right)$, also there are three bell membership function (MF) for every input parameter A2 (medium), A3 (far), and A1 (near), B1 (near), and B3 (far), and B2 (medium), C1 (near), C3 (far), and C2 (medium), D1 (negative), D3 (positive), and D2 (zero), correspondingly. The Takagi-Sugeno-type fuzzy control scheme IF-THEN instructions will then be built as below;

$\mathrm{IF}\left(x_{1}\right.$ is $A_{i}$ and $x_{2}$ is $B_{i}$ and $x_{3}$ is $C_{i}$ and $x_{4}$ is $D_{i}$

$\operatorname{THEN}\left(\right.$ steering angle $\left.\left(f_{n}\right)=p_{n} x_{1}+q_{n} x_{2}+r_{n} x_{3}+s_{n} x_{4}+u_{n}\right)$

The objective function sets are $A_{i}, \mathrm{~B}_{i}, C_{i}$ and $D_{i}$ of input parameters $x_{1}, x_{2}, x_{3}$ and $x_{4}$ correspondingly. Where $\mathrm{n}=1,2, \ldots .81$, and $\mathrm{i}=1,2,3$, and the linear values of output $f_{n}$ are $p_{n}, q_{n}, r_{n}$, $s_{n}$, and $u_{n}$, they are also called subsequent values, by varying those values we modify the outcome of the ANFIS controller. 


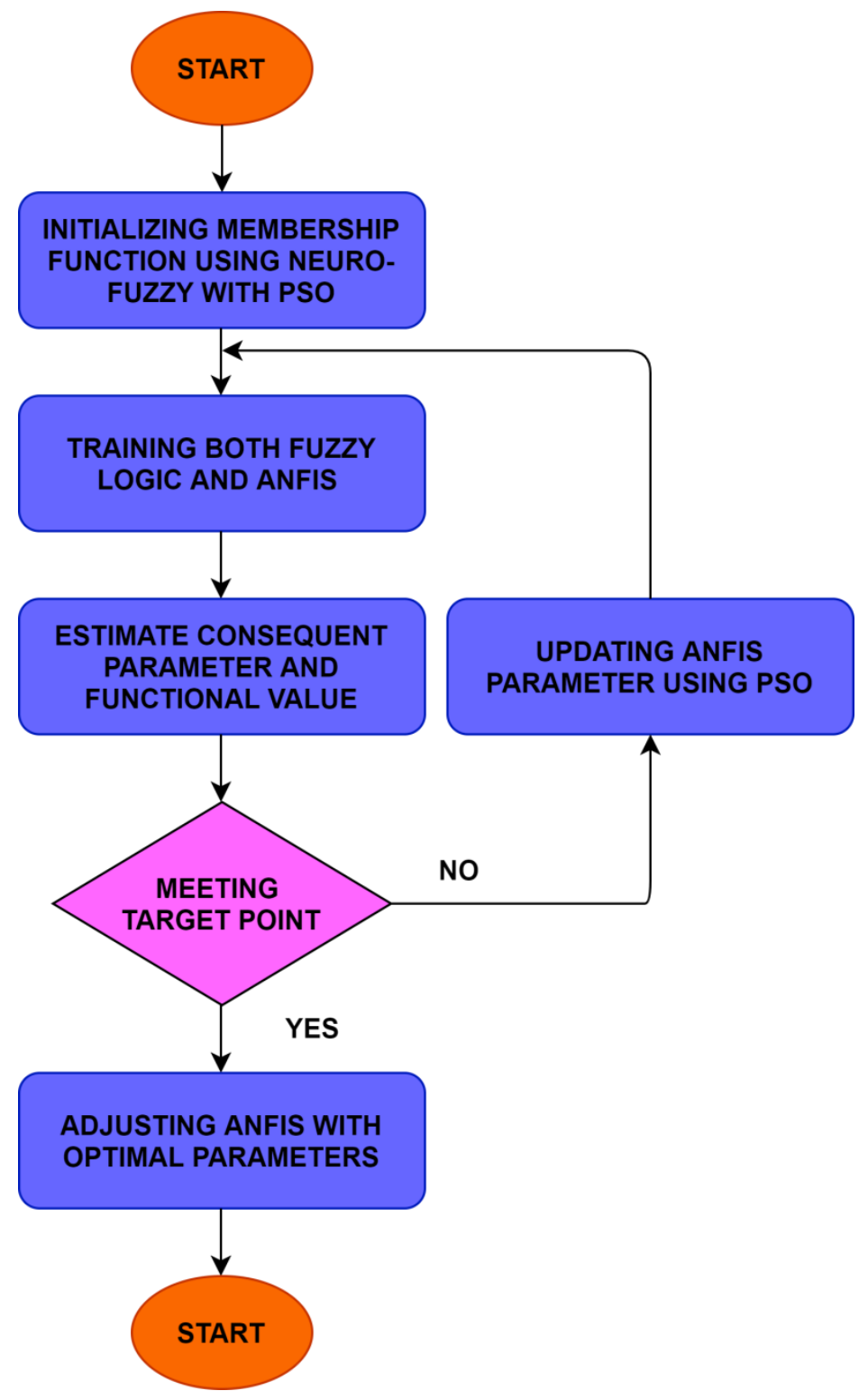

Figure 3: The proposed flowchart.

The proposed flowchart is shown in figure 3. We have defined three key reactive behaviors' in the present navigational system: one for goal searching, one is for wall tracking, another for avoiding impediments. The suggested ANFIS navigating controller contains several 12 sets of premises factors or non-linear variables $\left\{a_{i}, b_{i}, c_{i}\right\}$. The overall number of assumption variables that must be tweaked is $12 \times 3=36$. The range of rule base developed is $3 \times 3 \times 3 \times 3 \times 3 \times 3=81$, and that there are $81 \times 5=405$ numbers of subsequent variables or logistic variables $\left\{p_{n}, q_{n}, r_{n}, s_{n}, u_{n}\right\}$ to be taught in ANFIS to obtain the output signal. The PSO, as well as least-squares estimation (LSE) methods, are employed in ANFIS besides learning and updating the premises and conclusion variables in our suggested hybrid training approach. This ANFIS controller is designed to compute the objective function value of such a movable robot's steering angle created by the 
PSO methodology. Finally, by repetition of the PSO algorithm, the discrepancy between the output signal and the real training set can be lowered. In this case, the CS optimizer's objective function was specified while the Root Mean Square Error (RMSE) for the suggested ANFIS method.

$$
R M S E=\sqrt{\frac{1}{P} \sum_{i=1}^{P}\left(\theta_{i}-\theta_{i}^{\prime}\right)^{2}}
$$

If $i$ is the real steering angle, $i$ denotes the anticipated value of a robotic system produced again from the ANFIS-based PSO algorithm, and p denotes the overall number of occurrences. These repetitions will end whenever the RMSE value stays constant indicating that the PSO approach was unable to identify any superior variables to minimize the estimation error. The PSO-ANFIS hybrid approach is found to have a reduced failure rate than that of the traditional ANFIS approach. As a result, the range of variables included in every nest specifies an actual value of all available FIS basis variables. An excel file containing all 36 variables of ANFIS precursor variables has been created and maybe fetched by MATLAB while coding. The bell function was chosen also as a basic function for such fuzzy components, with the highest value of one as well as the lowest value of zero. The suggested PSO-ANFIS hybrid method can be utilized to lower the likelihood of getting stranded in local minima situations while also improving the ANFIS accuracy rate and universal searchability. It's been observed that now the improved hybrid method could save time while altering all ANFIS navigating controller variables.

\section{EXPERIMENTAL ANALYSIS}

The fuzzy inference methodology is used in the suggested technique of navigating in an uncertain place. It is made up of two attitudes (control system): the first one is utilized while there exists no barrier in front of the robots, and the other is activated whenever an impediment is identified. To assist comparability, the output of every type of simulation performed for both approaches are structured as shown below the route realized by every approach, and graphs of such necessary parameter values: Distance among the Robots as well as Total Distance Recognized by Robots (TDR), Navigation Time (ToN), and Targets during Navigate (DRT). 


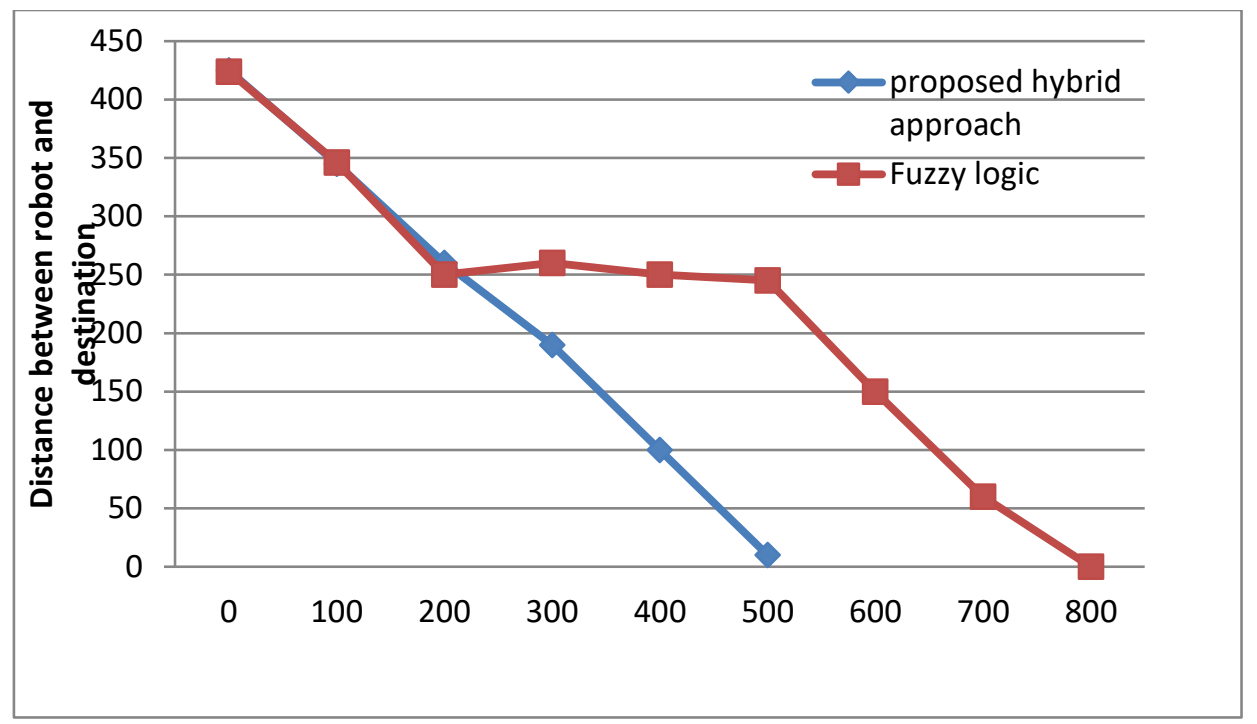

Figure 4: Target during Navigate (DRT) for both approaches for scenario 1.

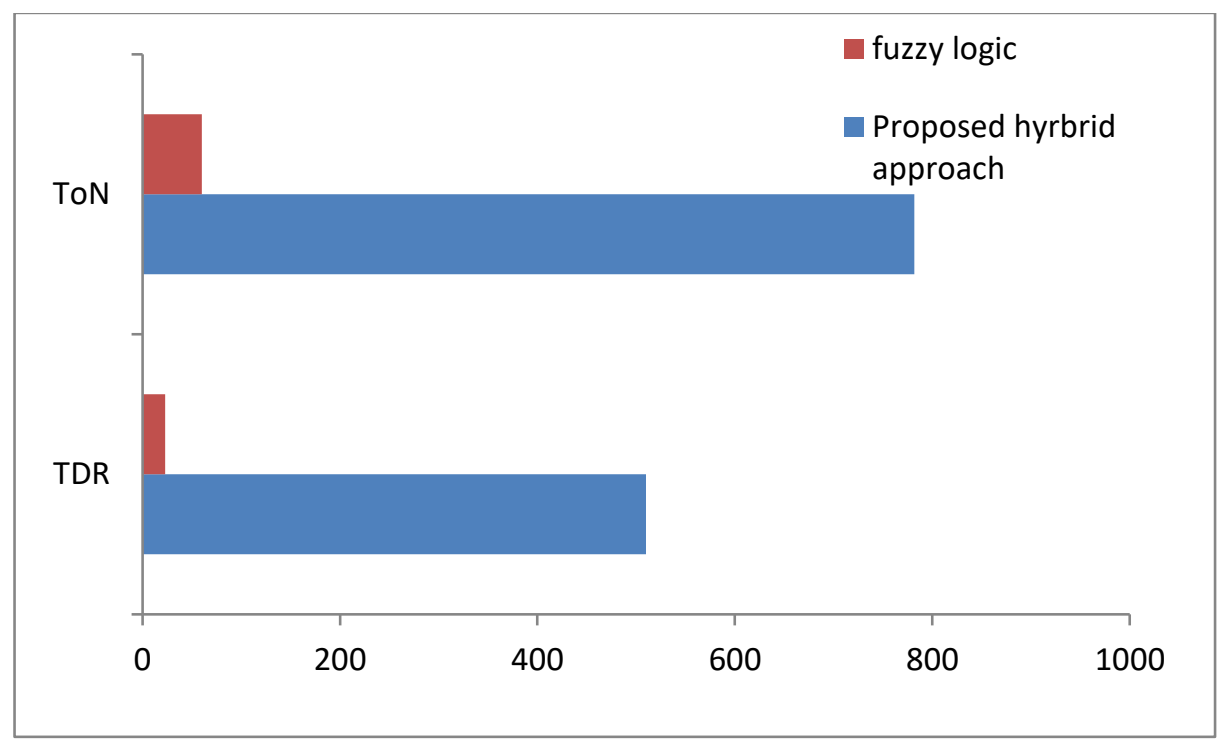

Figure 5: Comparison of both approaches with respect to ToN and TDR. 


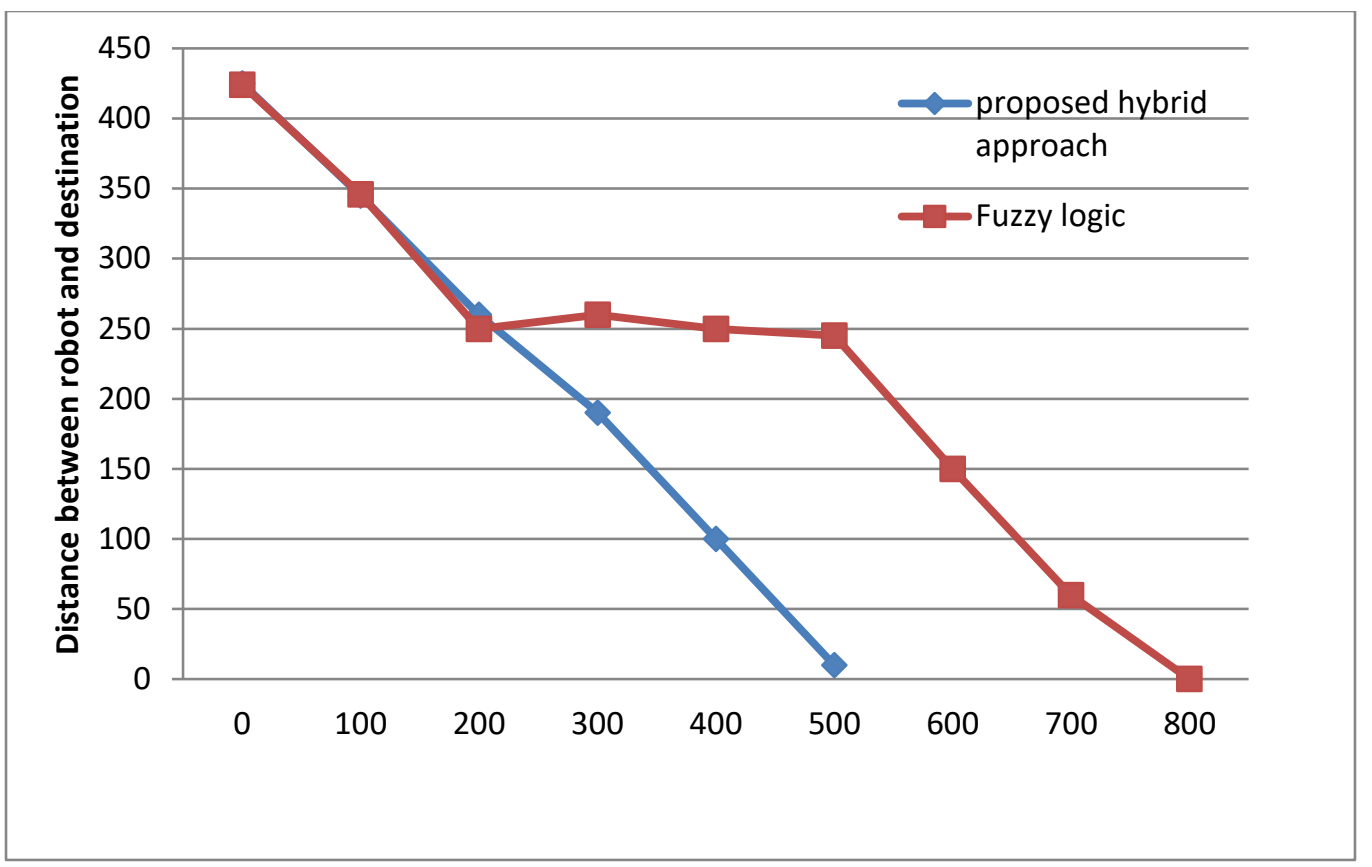

Figure 6: Target during Navigate (DRT) for both approaches for scenario 2.

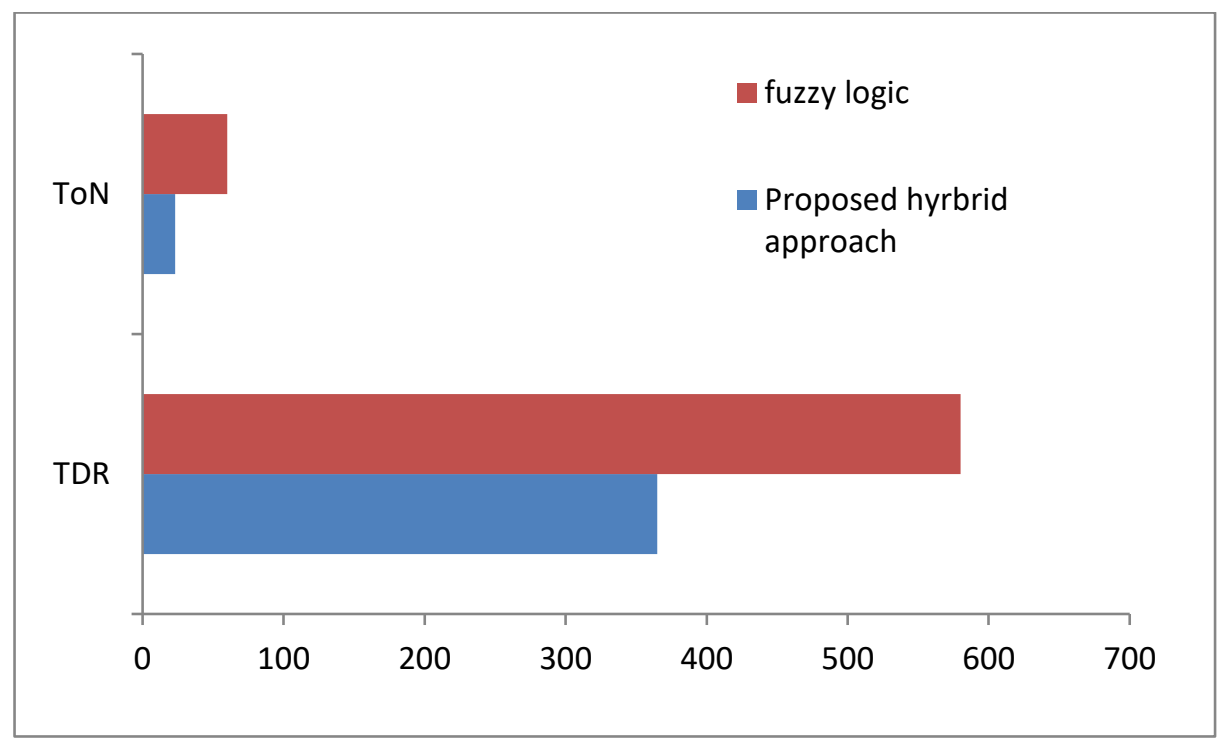

Figure 7: Comparison of both approaches with respect to ToN and TDR.

The proposed hybrid neuro-fuzzy technique outperforms a fuzzy logic technique and the representations of both techniques in terms of generating an optimum path. Whenever the robot employs our technique to achieve the destination it travels fewer distances, as well as takes lesser time to navigate Figure 4 and Figure 5 than a fuzzy logic approach. Figure 6 and Figure 7 illustrate the proximity among the robotic and also the destination throughout the navigational operation, demonstrating that our technique enables the robotic to be closer to the goal than that 
of the fuzzy inference system approach. Figures 4 and 5 illustrate the first scenario whereas Figure 6 and Figure 7 illustrate the next scenario.

\section{CONCLUSION}

This paper designs and tests a hybrid neuro fuzzy with PSO optimization for multi-robot navigation. A most utilized strategy for dealing with a navigational challenge in an unfamiliar place is neuro-fuzzy inference control. However, this approach suffering from limitations caused by the human building of objective function as well as the personally assigned linguistic values of outlets for type-0 fuzzy logic system. As a result, automated tweaking of membership value variables as well as fuzzy rule implications is necessary to achieve more effective guidance in respect of traveling duration and distance traversed. The neuro-fuzzy logic switching technique can efficiently combine these neural network and fuzzy logic characteristics; it is demonstrated by the simulated outcomes. Such architecture enables the robots to utilize action that corresponds to the condition in the surroundings (the existence or absence of impediments, as well as whether the surrounding is familiar or unfamiliar): The robot spends lesser time for navigating, saves calculation work among the present and target configurations, and seems to have an ideal pathway in most circumstances. Furthermore, simulation and practical studies show that the created navigational approach is adaptable to a wide range of challenging terrains.

\section{Ethics declarations}

Conflict of interest : The authors declare that they have no conflict of interest.

Human and animal rights : This article does not contain any studies with human participants or animals performed by any of the authors.

Funding : Not Applicable for this research

Availability of data and material : Not Applicable

Code availability : Not Applicable

Authorship contributions: The authors confirm contribution to the paper as follows: study conception and design: Dr. Dinesh Kumar Singh, Dr. Ananda Kumar Haldorai; analysis and interpretation of results: Dr. Shahanaz Ayub, Mr. Navneet Singh; draft manuscript preparation: Dr. Md. Zair Hussain, Dr. Mohd Ashraf. All authors reviewed the results and approved the final version of the manuscript. 


\section{REFERENCE}

1. Yan, F., Liu, Y.-S., \& Xiao, J.-Z. (2013). Path planning in complex 3D environments using a probabilistic roadmap method. International Journal of Automation and Computing, 10(6), pp. 525-533. doi: 10.1007/s11633-013-0750-9.

2. Yilmaz, N. K., Evangelinos, C., Lermusiaux, P. F. J., \& Patrikalakis, N. M. (2008). "Path planning of autonomous underwater vehicles for adaptive sampling using mixed integer linear programming. IEEE Journal of Oceanic Engineering, 33(4), pp. 522-537. doi:10.1109/JOE.2008.2002105.

3. Aghababa, M. P. (2012). "3D path planning for underwater vehicles using five evolutionary optimization algorithms avoiding static and energetic obstacles. Applied Ocean Research, 38, 48-62. doi:10.1016/j. apor.2012.06.002.

4. Hassani, I., Maalej, I., \& Rekik, C. (2018). Robot path planning with avoiding obstacles in known environment using free segments and turning points algorithm. Mathematical Problems in Engineering. doi:10.1155/2018/2163278.

5. Algabri, M., Mathkour, H., Ramdane, H., \& Alsulaiman, M. (2015). Comparative study of soft computing techniques for mobile robot navigation in an unknown environment. Computers in Human Behavior, 50, 42-56. doi:10.1016/j.chb.2015.03.062.

6. Rusu, C. G., Birou, I. T., \& Szoke, E. (2010) Fuzzy based obstacle avoidance system for autonomous mobile robot. IEEE International Conference on Automation Quality and Testing Robotics (AQTR) (pp. 1-6). Romania.

7. Hui, N. B., \& Pratihar, D. K. (2009). A comparative study on some navigation schemes of a real robot tackling moving obstacles. Robotics and Computer-Integrated Manufacturing, 25(4), 810-828. doi:10.1016/j. rcim.2008.12.003.

8. Selekwa, M. F., Dunlap, D. D., Shi, D., \& Collins, E. G. (2008). Robot navigation in very cluttered environments by preference-based fuzzy behaviors. Robotics and Autonomous Systems, 56(3), 231-246. doi:10.1016/j. robot.2007.07.006.

9. Pratihar, D. K., Deb, K., \& Ghosh, A. (1999). A genetic-fuzzy approach for mobile robot navigation among moving obstacles. International Journal of Approximate Reasoning, 20 (2), 145-172. doi:10.1016/S0888-613X (98)10026-9.

10. Abdessemed, F., Benmahammed, K., \& Monacelli, E. (2004). A fuzzy-based reactive controller for a non-holonomic mobile robot. ELSEVIER Robotics and Autonomous Systems, 47(1), 31-46. doi:10.1016/j. robot.2004.02.006.

11. Faisal, M., Hedjar, R., Al Sulaiman, M., \& Al-Mutib, K. (2013). Fuzzy logic navigation and obstacle avoidance by a mobile robot in an unknown dynamic environment. INTECH International Journal of Advanced Robotic Systems, 10(1), 1-7.

12. Dongshu, W., Yusheng, Z., \& Wenjie, S. (2011) Behavior-based hierarchical fuzzy control for mobile robot navigation in dynamic environment. IEEE International Conference on Control and Decision Conference (CCDC) (pp. 2419-2424). Mianyang, China.

13. Li, T. H. S., Chang, S. J., \& Chen, Y. X. (2003). Implementation of human-like driving skills by autonomous fuzzy behavior control on an FPGA-based car-like mobile robot. 
IEEE Transactions on Industrial Electronics, 50(5), 867-880. doi:10.1109/TIE.2003.817490.

14. Li, T. H. S., Chang, S. J., \& Tong, W. (2004a). Fuzzy target tracking control of autonomous mobile robots by using infrared sensors. IEEE Transactions on Fuzzy Systems, 12(4), 491501. doi:10.1109/ TFUZZ.2004.832526.

15. Ayari, E., Hadouaj, S., \& Ghedira, K. (2010) A fuzzy logic method for autonomous robot navigation in dynamic and uncertain environment composed with complex traps. IEEE 5 th International Multi-conference on Computing in the Global Information Technology (pp. 18-23). Valencia, Spain.

16. Antonelli, G., Chiaverini, S., \& Fusco, G. (2007). A fuzzy-logic-based approach for mobile robot path tracking. IEEE Transactions on Fuzzy Systems, 15(2), 211-221. doi:10.1109/TFUZZ.2006.879998.

17. Zhu, A., \& Yang, S. X. (2007). Neurofuzzy-based approach to mobile robot navigation in unknown environments. IEEE transactions on systems. Man, 37(4), 610-621.

18. Godjevac, J., \& Steele, N. (1999). Neuro-fuzzy control of a mobile robot. Elsevier Neurocomputing, 28(1), 127-143. doi:10.1016/S0925-2312(98)00119-2.

19. Al Mutib, K., \& Mattar, E. (2011) Neuro-fuzzy controlled autonomous mobile robotics system. IEEE 13th International Conference on Modelling and Simulation, UK, p. 1-7.

20. Li, W., Ma, C., \& Wahl, F. M. (1997). A neuro-fuzzy system architecture for behaviorbased control of a mobile robot in unknown environments. Fuzzy Sets and Systems, 87(2), 133-140. doi:10.1016/S0165- 0114(95)00015-1.

21. Kim, C. J., \& Chwa, D. (2015). Obstacle avoidance method for wheeled mobile robots using interval type-2 fuzzy neural network. IEEE Transactions on Fuzzy Systems, 23(3), 677-687. doi:10.1109/TFUZZ.2014.2321771.

22. Zhang, N., Beetner, D., Wunsch, D. C., Hemmelman, B., \& Hasan, A. (2005) An embedded real-time neuro-fuzzy controller for mobile robot navigation. IEEE International Conference on Fuzzy Systems (pp. 319-324). USA.

23. Marichal, G. N., Acosta, L., Moreno, L., Mendez, J. A., Rodrigo, J. J., \& Sigut, M. (2001). Obstacle avoidance for a mobile robot: A neuro-fuzzy approach. Fuzzy Sets and Systems, 124(2), 171-179. doi:10.1016/ S0165-0114(00)00095-6.

24. Ma, X., Li, X., \& Qiao, H. (2001). Fuzzy neural network-based real-time self-reaction of mobile robot in unknown environments. ELSEVIER Mechatronic, 11(8), 1039-1052. doi:10.1016/S0957- 4158(00)00061-1.

25. Baturone, I., Gersnoviez, A., \& Barriga, A. (2014). Neurofuzzy techniques to optimize an FPGA embedded controller for robot navigation. In ELSEVIER Applied Soft Computing21 (pp. 95-106). Appl. Soft Comput.

26. Imen, M., Mohammad, M., \& Shoorehdeli, M. A. (2011) Tracking control of mobile robot using ANFIS. IEEE International Conference on Mechatronics and Automation (pp. 422427). China. 
27. Ganapathy, V., Yun, S. C., \& Ng, J. (2009) Fuzzy and neural controllers for acute obstacle avoidance in mobile robot navigation. IEEE/ASME International Conference on Advanced Intelligent Mechatronics (pp. 1236-1241). Singapore.

28. Zhao, T., \& Wang, Y. A. (2012) Neural-network based autonomous navigation system using mobile robots. IEEE 12th International Conference on Control, Automation, Robotics \& Vision (ICARCV) (pp. 1101-1106). China. 